\title{
MULTIPLE SINGLE BUNCH EXTRACTION TO THE AGS SWITCHYARD *
}

\author{
K.A. Brown, L. Ahrens, D. Gassner, J.W. Glenn, T. Roser, G. Smith, N. Tsoupas, \\ W. van Asselt, K. Zeno, \\ Brookhaven National Laboratory, Upton, New York 11973, USA
}

\begin{abstract}
In this report we will describe the multiple single bunch extraction system as utilized to deliver beams to the Brookhaven's Alternating Gradient Synchrotron (AGS) switchyard area. We will describe modifications of the AGS switchyard, necessary to allow it to accept bunched beam, and results of the first commissioning of this system. The AGS Switchyard has for many years been used to simultaneously deliver (unbunched) resonant extracted beam to a set of fixed target experiments. In order to accommodate new fixed target experiments which require bunched beams, a method of sending the bunched beams to the AGS Switchyard was required. In addition, by using the AGS switchyard instead of the upstream section of the Brookhaven's Relativistic Heavy Ion Collider (RHIC) injection line the accelerators can be reconfigured quickly and efficiently for filling RHIC. We will present results of the commissioning of this system, which was done in January 2001.
\end{abstract}

\section{INTRODUCTION}

The AGS has been delivering fast beam pulses to various experiments for decades. In 1996 a new fast extraction system was put in place and commissioned [1]. This new system was primarily designed to do bucket to bucket bunch transfers from the AGS to RHIC. This multiple single bunch extraction system has since been utilized to deliver high intensity protons to the production target for G-2 (G-2) [2], gold ions and polarized protons to RHIC [3, 4], as well as to deliver various intensity and various energy single bunch beams to smaller experiments setup on a beam-line which branches off the AGS to RHIC transfer beam-line (ATR).

To allow performing the various smaller fast beam experiments simultaneously with RHIC operations we needed an alternative location to deliver the fast beam pulses. The AGS switchyard is an ideal location, since all the infrastructure is in place for setting up fixed target experiments. By running the AGS in a different configuration (including operating at a different working point) we have found we can efficiently deliver fast beam pulses using the existing fast kicker magnet used for sending beam to G-2 and ATR. The modifications we needed to make to the AGS switchyard were modest. We needed to instrument the switchyard for fast beam operation and we needed to be optically

\footnotetext{
${ }^{*}$ Work performed under the auspices of the U.S. Dept. of Energy.
}

matched to the AGS, which was done with the existing matching section quadrupoles.

An important piece to this system is the ability to perform a "context switch" from one operation (e.g., sending beam to G-2) to one which delivered beam to the switchyard, and back again. The AGS has had the ability to perform such context switches for many years. Using this system we were able to "steal" beam pulses away from the G-2 cycle on a pulse to pulse basis. In addition there was a need to switch from sending high intensity proton bunches to G-2 to sending low intensity slowly extracted protons to the switchyard, while retaining the fast beam to switchyard setup. To do this we have made use of the new RHIC sequencer system [5], which allows automated changing of the state of a particular subsystem (e.g., changing the rigidity of the beam line). This ability to switch modes allowed us to switch on a shift by shift basis, from running high intensity proton bunches to one beam line in the switchyard to running low intensity slowly extracted beam to another beam line in the switchyard. The combination of performing context switches and of performing mode switches gives the Brookhaven hadron accelerator complex an unprecedented high degree of flexibility, making simultaneous operation of many different experiments possible.

\section{DESCRIPTION OF EXTRACTION PROCESS}

To perform (conventional) multiple single bunch extraction to RHIC we employ a combination of an open "C" type fast kicker magnet with a thick septum magnet. The fast kicker provides a $1.6 \mathrm{mrad}$ kick, which will displace a beam as large as $35 \mathrm{~mm}$ across the $1 \mathrm{~cm}$ septum of the thick septum magnet. A more detailed description of this system is given in reference [6]. To extract beam into the AGS switchyard the kicked beam must survive traversing 11 out the 12 AGS superperiods (or essentially one full turn). To get the largest displacement at the thin and thick septa normally used for resonant extracted beam, the horizontal tune must be dropped to very near the $1 / 2$ integer. Figure 1 shows the amount of displacement at the thin and thick septa as a function of horizontal tune.

Kicking beam across the thin septum is easily done since the septum is very thin $(0.76 \mathrm{~mm})$ relative to the beam size. The more difficult task is clearing the beam past the thick septum $(16 \mathrm{~mm})$. By using the combination of the fast kicker, the deflection due to the thin septum, and operat- 


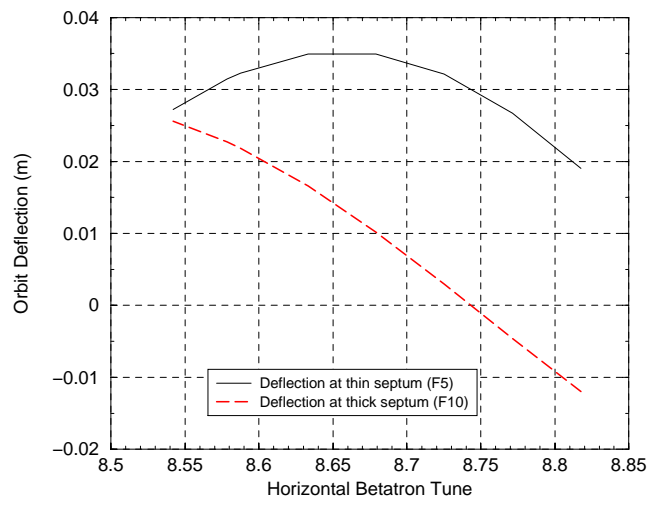

Figure 1: Central trajectory displacement as a function of horizontal tune at the location of the thin and thick septa, for a $1.8 \mathrm{mrad}$ kick at $24 \mathrm{GeV}$

ing at a very low horizontal tune, we can get a 25 to 30 $\mathrm{mm}$ beam across the $16 \mathrm{~mm}$ thick septum. Without the thin septum this would not be possible, since the largest deflection at F10 is on the order of 20 to $25 \mathrm{~mm}$. The size of the beam that can be extracted is smaller than for the normal fast beam channel, and this limits the beam emittance to approximately 100 pi-mm-mrad $99 \%$ normalized (ignoring momentum dispersion).

\section{FY01 COMMISSIONING RESULTS}

At the end of January, 2001 the system was commissioned and both the neutron spallation target experiments [7] and the muon collider target test experiments [8] were able to complete an aggressive program, while simultaneously running for the G-2 experiment.

The extraction system performed very well and we were able to cleanly extract as much as $4.5 \times 10^{12}$ protons in a single bunch. We also ran with multiple bunch extraction. In this case we ran as high as $3.5 \times 10^{13}$ in 12 bunches.

Figure 2 shows the orbit deformation required to put the beam into the fast kicker and near enough to the two septum magnets such that when kicked, the beam is displaced across the septa. Included on this figure are beam position measurements from the AGS orbit acquisition system. Figure 3 shows the trajectories at the locations of the thin and thick septa. Of note is the vertical sextupole at the F7 location. In order to clear the thick septum cleanly we found we had to run the vertical sextupoles at $-175 \mathrm{amp}$, which caused the vertical chromaticity to be negative.

\section{BEAM INTENSITY LIMITATIONS}

During commissioning we quickly found that we were limited in the single bunch beam intensity to about $5.5 \times 10^{12}$ protons. The beam losses increased on all devices with intensity, but increased most non-linearly at the kicker location. This is suggestive of vertical blow-up. As noted in the previous section the vertical chromaticity was forced to be negative to cleanly get past the thick septum

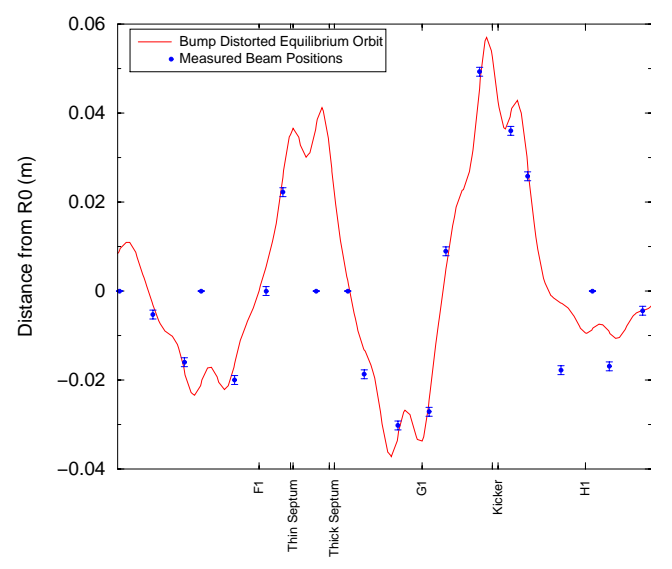

Figure 2: Measured orbit points and the predicted orbit around the kicker and septa locations.

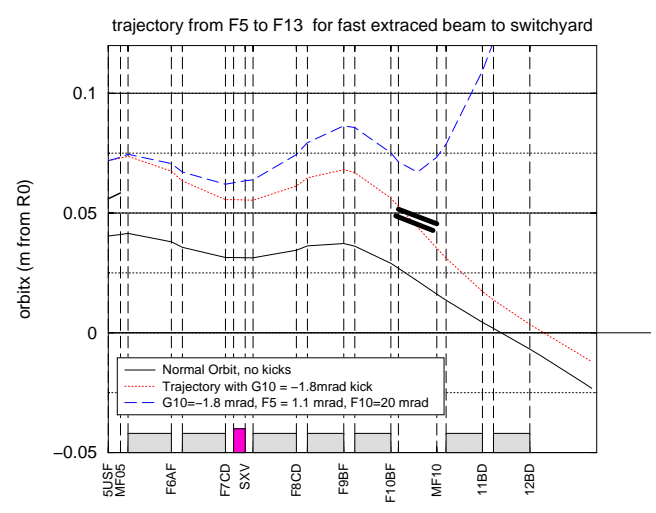

Figure 3: Central trajectories of kicked and unkicked beam. The location of the thin and thick septa are marked on the figure. The location of the vertical sextupole at F7 is marked in color. The solid black line is the unkicked central beam orbit. The red dotted line is the displaced orbit due to the G10 kicker. The dashed blue line is the final trajectory including the thin and thick septa bends.

(even at low intensities). It has been suggested that a possible reason is a local effect due to the F7 vertical sextupole. Nevertheless, a side effect of this is the vertical chromaticity is negative, which could cause higher sensitivity to some instabilities (e.g., transverse head-tail instability) [9]. Figure 4 shows the losses on the kicker (G10), the thin septum (F5), and the thick septum (F10), as a function of intensity. The straight lines are drawn to guide the eye. There is a clear change in the losses just above $4.5 \times 10^{12}$ protons/bunch, which is what we expect if there is an emittance blow-up due to instabilities. Figure 5 shows the extracted beam intensity as a function of the internal intensity. Above $6 \times 10^{12}$ protons (internal) we begin to see diminishing returns. Through further adjustments of machine parameters we were able to get up to $5.5 \times 10^{12}$ protons extracted, but the general character of this curve didn't change.

In an effort to achieve higher single bunch intensities we explored various modes of operation. Initially the AGS was 


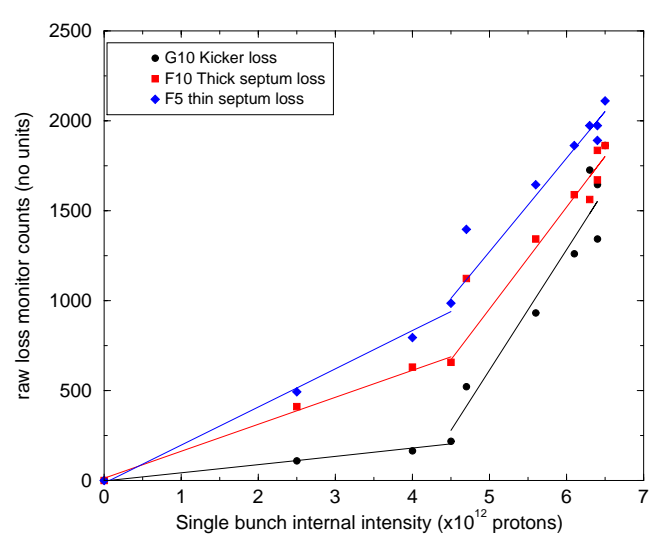

Figure 4: Beam losses versus intensity (loss monitors have roughly equal response to a given amount of lost beam)

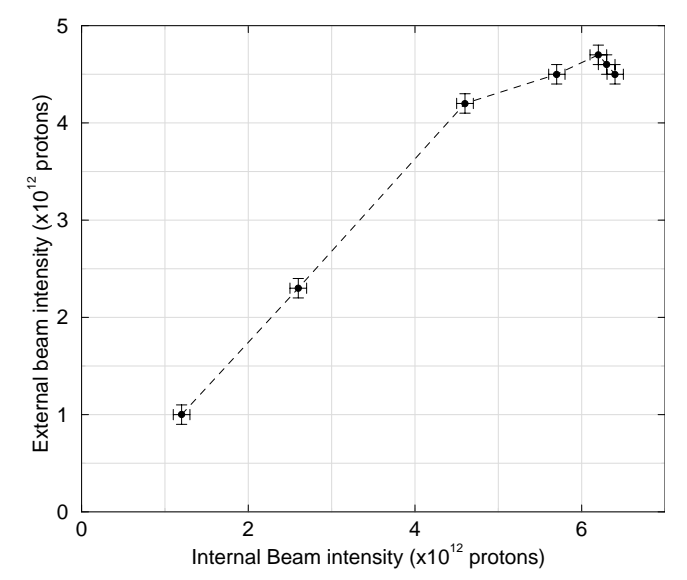

Figure 5: extracted beam intensity versus internal intensity

configured to accept beam from the Booster into $\mathrm{h}=6$ buckets. The 6 bunches were then split adiabatically into $h=12$ buckets and then accelerated. This allowed us to operate the Booster on $\mathrm{h}=1$, using 6 transfers to the AGS. In this mode of running the AGS has achieved very high beam intensities [10]. For the normal fast beam to switchyard setup we mistuned the bunch split to put all of the beam into a single $\mathrm{h}=12$ bucket.

To avoid losses that normally occur on very long injection porch we attempted to inject directly into an $\mathrm{h}=12$ bucket (single bunch) and then accelerate it. This caused the longitudinal emittance to become much smaller than normal, causing difficulties going through transition. In the end we didn't achieve much better than in the previous mode of running. We also attempted to run on $\mathrm{h}=6$ through the entire cycle (no bunch split). In principle this should have given us a factor of 2 , but collective effects caused problems at extraction time. We did achieve internally very high intensity bunches, but at best extracted $5.5 \times 10^{12}$ protons in one bunch, with relatively high losses at extraction.

\section{INSTRUMENTATION}

The most significant change we made to the AGS switchyard was to install pulsed beam instrumentation. We added three current transformers, one at the most upstream part of the switchyard, inside the matching section quadrupoles and two more, just upstream of the experimenter target stations.

\section{FUTURE MODES OF RUNNING}

To satisfy the needs of the fast beam experiments we need to get to higher single bunch intensities. Our goal is $1.6 \times 10^{13}$ in a single bunch. We are looking into a number of ways of getting to these kind of intensities. All the techniques that we would employ require doing bunch merging on the extraction flattop. Future studies will include bunch merging gymnastics, higher bunch dilutions (to avoid collective effects), and chromaticity studies.

\section{CONCLUSIONS}

Through the use of an existing fast extraction system we have been able to dynamically reconfigure the AGS to kick bunches from the G-2 line to the AGS switchyard. This has allowed the AGS to run multiple simultaneous experiments and gives unprecedented flexibility to the accelerators at BNL.

\section{REFERENCES}

[1] M.Tanaka et al., "The AGS Accelerator Complex with the New Fast Extraction System.”, Proceedings of the 1995 Particle Accelerator Conference, Dallas, Texas (IEEE, Piscataway, NJ, 1996), Vol.3, pp. 1930-1932.

[2] R.M. Carey et al., "New Measurement of the Anomalous Magnetic Moment of the Positive Muon", Phys. Rev. Lett. 82, 1632-1635 (1999).

[3] D. Trbojevic et al, "Commissioning of the Relativistic Heavy Ion Collider", These proceedings.

[4] W. MacKay et al, "Commissioning and future plans for polarized protons in RHIC", These proceedings.

[5] J. van Zeijts et al, "The RHIC Sequencer", These Proceedings.

[6] N.Tsoupas et al., "Beam Parameters for the AGS Synchrotron during Fast Beam Extraction”, These proceedings.

[7] T.A. Gabriel et al, "Target Systems Overview for the Spallation Neutron Source", Nuclear Technology, pp.49-60, Vol.32, October 2000.

[8] H. Kirk et al, "Target Studies with BNL E951 at the AGS.", These Proceedings.

[9] B. Zotter, in Handbook of Accelerator Physics and Engineering, edited by A. Chao and M. Tigner (World Scientific, Singapore, 1999), p.117

[10] L. Ahrens et al., "High Intensity Performance of the Brookhaven AGS" Proceedings of the 1999 Particle Accelerator Conference, New York, New York (IEEE, Piscataway, NJ, 2000), pp. 614-616. 